\section{PRECISE PROBING OF PERIO POCKETS}

The pa-on from Plandent is the first fully automatic perio probe for the precise detection of perio pockets.

With consistent calibrated pressure of 20g, pa-on will give a true and reliable reading every time. The pa-on charts immediately without the need for a second person to record the measurements and with acoustic and visual feedback you always know exactly where you are in the mouth. The small diameter of the probe makes this unique measuring device less invasive and more comfortable for the patient.

$\mathrm{Pa}$-on measures recession, bleeding, plaque as well as other extra features that can be directly included during the screening at a touch of a button on this software based wireless system. Your favourite perio status scheme can be programmed onto pa-on and it's easily integrated in byzz and all other existing software systems by placing the probe into its docking station. The measurements are immediately available on the screen for clarification and patient education.

With hygienic one way tips, pa-on is guaranteed to make perio pocket measuring a pleasure.

For more information call Plandent directly on 0500500322.

\section{CORD AND BATTERY-FREE CURING LIGHT}

Kerr has launched the Demi Ultra LED Ultracapacitor Curing Light System. Representing the latest technological advancement in dental curing from the Kerr Demi brand, it is the first curing light to free dentists from batteries and cords, while offering the unmatched performance and reliability of a Demetron curing light.

The product also has a non-degrading power source, rapid recharge in 40 seconds or fewer, uniform depth of cure, low heat generation without compromising cure time (CURE technology), and simple and intuitive operation and maintenance. The unit can be swapped should failure occur and the product offers warranty extension and accidental damage cover.

For more information, call Kerr on 01733892292 or visit www.kerrdental.co.uk.

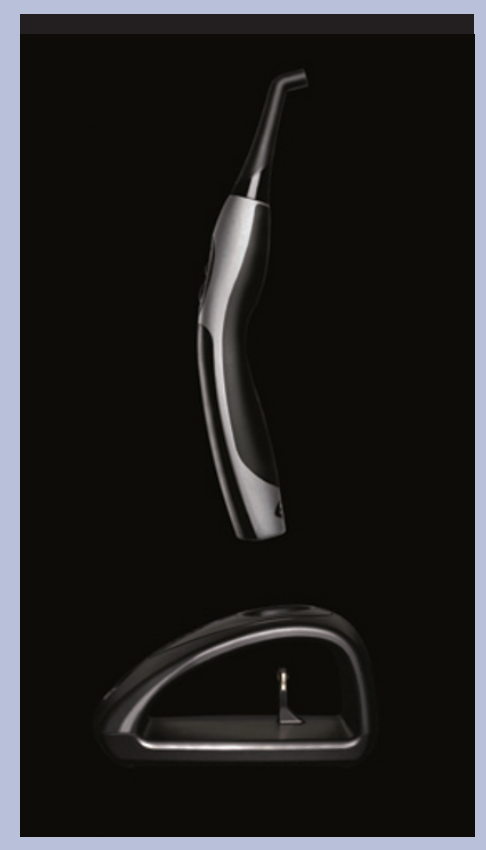

\title{
GENUINE INTEGRATED IMPLANTOLOGY
}

Sirona Dental Systems has introduced ground-breaking solutions for integrated implant planning and treatment that combine the digital impression scanning and design capabilities of CEREC with the diagnostic accuracy of Sirona's 3D X-ray units. Combine CEREC digital impressions and computer-

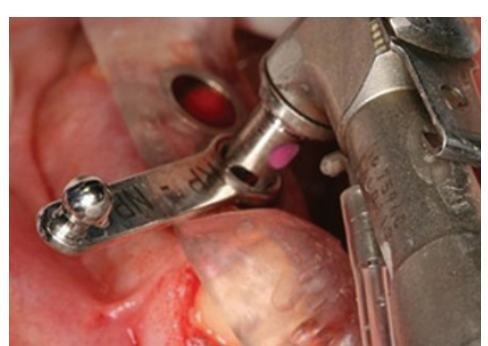

aided design with the diagnostic security of Sirona's 3D X-ray systems for genuine integrated implantology. It is now possible to perform surgical and prosthetic planning simultaneously - and then implement this plan precisely using a surgical guide.

This is ideal for all practitioners who would like to place more implants in future - or those who want to provide implant therapy for the first time with higher clinical security as a result of the surgical guides and perfect documentation of the implant job. You save time and money with fewer treatment sessions and achieve significant efficiency gains with a streamlined workflow. You can also show your patients what the final result will look like, leading to a higher treatment plan acceptance.

For further information call Sirona Dental Systems on 08450715040 or email info@sironadental.co.uk.

\section{ANOTHER IMPLANT RANGE BREAKTHROUGH}

Straumann has developed another breakthrough with a new range of implants featuring an advanced combination of Roxolid material, SLActive surface technology and the NEW Loxim transfer piece for simplified handling.

Now featuring across all diameters, Straumann's new generation of Roxolid SLActive implants provide clinicians with all the benefits of Roxolid's exceptional material strength together with the excellent osseointegration properties offered by SLActive surface technology.

Loxim, the new pre-mounted, self-retained transfer piece, enables both clockwise and counterclockwise rotation for one step implant insertion.

Together, these materials ensure greater confidence in all cases for the entire implant team, successfully enabling the treatment of more patients.

Call 01293651230 or visit www.straumann.com.

\section{DEVELOP YOUR NEW BUSINESS STREAM}

Learn how to restore dental implants with confidence by taking part in an Esthetic Alliance Program from Nobel Biocare. Dentists are supported in all aspects of developing this new business stream, from marketing their service to sourcing patients and developing networks with partner surgeons and laboratories.

The tuition is provided by highly experienced surgeons and facilitated by Nobel Biocare. Each session takes place in a small group setting to stimulate interactive learning at your own pace. Call 02087563300 to find out more. 